\title{
Evaluation of Thailand's Drug Abuse Resistance Education Project (DARE) Provincial Police Region 1
}

Apasiri Suwannanon, Graduate School, Suan Dusit Rajabhat University, Thailand Abstract

Aim of this research is to study the factors affecting the problems and obstructions of the Drug Abuse Resistance Education (DARE) project. This research evaluates project administration, the processes of cooperation among key persons, success of gaining knowledge, positive attitudes and behaviour of students after rehabilitation. The research also suggests methods to improve and develop the operation of the project in the area of the Provincial Police Region 1.

Research methodologies were qualitative and quantitative and the participants were interviewed in detail about their field observations as well as their participation in discussions.

The key success factors included the support of the heads of relevant organisations. Positive aspect of the DARE project is that it can prevent youths from taking drugs for some time. The negative aspects were the lack of understanding of the police towards project objectives. The suggestions for improvements mostly included providing mobile standard equipment for DARE police and the operation of DARE youth camps in schools, establishment of DARE police clubs and the operation of DARE police groups, which included the commissioned officers and the warrant officers, and curriculum improvement to suit Thai culture and drug situations.

\section{Introduction}

Thailand still faces the problem of illicit drug trafficking and smuggling from the Golden Triangle and neighbouring countries. 'Methamphetamine' still remains a major seized drug. Royal Thai Government's approach is to solve drug problems in a comprehensive and systematic manner, from prevention and suppression to rehabilitation of drug addicts. Thailand's Drugs Control Strategy is prevention. For the 'General group', it will launch anti-drugs campaign and provide drug information in various forms to give knowledge about drugs. For the 'Sensitive group', it will perform surveys and collect information, carry out anti-drugs activities and monitor sensitive locations. For the 'Educational group' it will establish a core group (teachers, parents, students) in educational institutions and communities to watch drugs abusers and develop teaching and learning curriculum to prevent taking drugs. 
The Drug Abuse Resistance Education (DARE) project or DARE police is one of the major drug prevention projects which focus on the youth target groups organized by police officers. DARE project gives kids life skills they need to avoid getting addicted to drugs, avoid gangs and also violence. DARE was found in 1983 by the Police Academy in Los Angeles and has proved successful in preventing drug abuse and other problems related to it. DARE is now implemented in schools and in more than 43 countries around the world. ${ }^{1}$ In the United States, DARE is one of the most widely used substance abuse prevention programmes, targeted at school-aged youth. In recent years, DARE has been the country's largest single school-based prevention programme in terms of federal expenditure, with an average of three quarters of a billion dollars spent on its provision annually. ${ }^{2}$ DARE operates in about 80 percent of all school districts across the United States and in many foreign countries. $^{3}$

DARE's primary mission is to provide children with information and skills they need to lead drug and violence free lives, by educating them about drug menace through programmes at elementary, middle and high school levels. ${ }^{4}$ Lesson plans for DARE focus on four major areas.

- to provide accurate data about using drugs, alcohol as well as tobacco.

- to teach students decision-making skills.

- to show students how to recognize and resist peer pressure, and,

- to inform students about positive alternatives to drug use.

DARE officers are working with children to raise their self-esteem, teach them how to make decisions on their own, and help them identify positive alternatives to drugs. By role-playing, the DARE curriculum emphasises the negative consequences of drug use and reinforces skills to resist peer pressure. Moreover, DARE is a cooperative effort by the police, schools, parents, and communities - all four working together to help children make right choices to abstain from drug use. ${ }^{1}$ One of the unique features of DARE is the use of uniformed police officers as instructors assigned to classrooms.

The DARE curriculum is designed to be taught by police officers whose training and experience give them the background necessary to answer sophisticated questions, often posed by young students about drugs and crimes. ${ }^{1}$ Prior to entering the DARE program, officers underwent 80 hours of special training in areas such as child development, classroom management, teaching techniques and communication skills. Forty hours of additional training were provided to DARE instructors to prepare them to teach the high school curriculum on prevention of drug abuse. 
For the follow-up and evaluation of DARE, it was found that there was a significant relationship between earlier DARE participation and less use of illegal, more deviant drugs (e.g., inhalants, cocaine, LSD) in a development sample, and not in a validation sample. ${ }^{5}$

DARE project was founded in Thailand in 1999. The Office of Narcotics Control Board of Thailand (ONCB) is considered as the office of the DARE project, which supported the national strategy of solving drug problem in high risk youth groups. The objective of the project is to educate youth about the resistance to drugs, the negative action of gangs and anti-violence in youth groups. The ONCB has given financial support to the group of researchers from Suan Dusit Rajabhat University of Thailand to organise the research project to evaluate the DARE project in the area of Provincial Police Region 1, in 2010.

\section{Objectives}

1. To study the models of the DARE project in Thailand and foreign countries

2. To study factors affecting problems and obstructions for successful implementation of the project

3. To evaluate project administration, cooperation of DARE police with the schools, provincial organisations as well as the parents, the success of raising knowledge and positive attitudes, behaviour of the students and to prevent them from drug abuse

4. To suggest ways to improve and to develop the operation of the DARE project in the area of Provincial Police Region 1

\section{Methods}

Research methodologies were qualitative and quantitative. To gather data for qualitative methodologies, in-depth interviews and field observations were conducted. The sample groups were the head administrator, the administrators of the project, resources persons of the DARE project, teachers, parents, local administrators and DARE police in the area of Provincial Police Region 1 (the central part of Thailand), who were involved in the project, out of a total of 64 persons. The researchers also used the focus group discussions of concerned persons to comment and to confirm the result of the research from 12 persons. For the quantitative approach, the researchers used questionnaires to gather data from 360 students involved in the project, in the area of Provincial Police Region 1. 


\section{Results}

\section{Key factors of success}

In the operation, the project was supported by the head administrators and the head of the police stations. Continuous training for knowledge and skill development of DARE police also helped the programme. It included voluntary participation of DARE police, the support and cooperation from administrators and teachers in schools, the continuous support from the governors and also local administrators.

\section{Problems and obstructions}

In the operation of the project, there were many obstacles such as instability of the status of police of the area, lack of financial support for the operation, administration and special activities of the project, as well as lack of public support for the project in the province. Seeking the support from relevant people might be seen as a benefit. The length of the DARE curriculum had to be covered in a short period of time. Lack of parentchildren relationships, lack of DARE police officers in some areas and the length of the DARE curriculum which was not suitable for the current drug situation in Thailand were some problems.

\section{Positive aspects}

The DARE project can prevent youth from taking drugs for some time. DARE police officers in uniform can be satisfied that they have educated students about risks of taking dangerous drugs. School administrators as well as teachers can participate in the project to inform students such risks and to prevent students taking drugs. Students can also inform their friends and parents the necessity to reject such drugs. Students have rational thinking and were interested in studying in DARE classes. The project was worthwhile compared to the cost of the drug treatments and rehabilitation. Many people are interested to see that the DARE project continue and expand, in spite of its ineffectiveness and even harm to students. About two million baht is spent for the programme each year, and thousands of police officers are assigned to educate students about the risks of taking dangerous drugs.

\section{Negative aspects}

Police officers do not understand the objectives of the project. Some details of the curriculum were not suitable for Thai culture and society. Lack of financial support for learning equipment and teaching and the short duration of teaching will make the students become the risk group in taking drugs. Lack of continuous support and cooperation from local administration is also a negative aspect. There are many reasons for the 
remarkable ineffectiveness of the DARE project. For example, it is based on out-dated theories of learning and human behaviour and it failed to distinguish between legal substances and illegal drugs. It views all use as abuse and it presents a view of substance use inconsistent with what most students see in their environment.

\section{Suggestions for urgent implementation}

Suggestions for urgent operation are organising mobile standard equipment for DARE police, curriculum improvement to suit Thai culture and the drug situation in Thailand. When increasing financial support is given for the project operation, the budget should be sent directly to the key officers who worked in the area. Increase in the cadres of DARE policemen and police women in every area, integration of DARE curriculum in the official curriculum of primary and secondary schools and the development of continuous follow-up and evaluation operation of the project are some other suggestions.

\section{Suggestions for project operation and curriculum}

Suggestions for project operation and curriculum are operation of DARE youth camps in schools, establishment of a DARE police clubs, and operation of DARE police groups which included Commissioned Officers and Warrant Officers. Other suggestions for the success of the project are separation of DARE police training in the operation and skills in teaching practical courses, finding financial resources to support the project, preparing an official directory of DARE students and DARE, expansion of DARE operation in government and private schools, having a website of DARE project for the members concerned, promotion of a strong cooperation among DARE police, teachers in schools, local administration and with relevant organizations.

\section{Conclusion}

The DARE project or DARE police is one of the major drug prevention projects, which stress on the youth target groups and was organized by the police officers. The ONCB considers the DARE project as one of its drug prevention projects, which supports the nation's strategy of solving the drug problem among youth. The objectives of the research were to study the models of the DARE project in Thailand and foreign countries, to study the factors affecting the problems and obstructions of the project, to evaluate the project administration and the role of DARE police officers and their cooperation with school administrations, provincial organisations and parents, success of organising awareness campaigns, positive attitudes to prevent students taking drugs, and to suggest ways to improve and to develop the operation of the DARE project in the 
area of the Provincial Police Region 1. Research methodologies are both qualitative and quantitative; the qualitative methodology used in-depth interviews and field observation methods. Sample groups were the head administrator, the administrators of the project, and resources persons of the DARE project, teachers, parent, local administrators and DARE police in the area of Provincial Police Region 1. The present study showed that it is not a $100 \%$ successful program.

\section{References}

1 Dover Police DARE (2006). History and Overview of DARE. News Release DARE News www.doveryouth.com Accessed on December, 2012.

2 McNeal RB, Hanson WB ( 1995 ). An examination of strategies for gaining convergent validity in natural experiments : DARE as an illustrative case study : 141-158

3 DARE America www.DARE.com Accessed on December 2012

4 US General Accounting Office (2003). Youth Illicit Drug Use Prevention: D.A.R.E. Long-Term Evaluations and Federal Efforts to Identify Effective Programs, Jan 16, 2003. (GAO-03-172R)

5 Dukes RL, Stein JA, Ullman JB. Long-term impact of Drug Abuse Resistance Education (D.A.R.E.). Eval Rev. 1997 Aug;21(4):483-500 